\title{
COVID-19-Related Anxiety and Its Association with Dietary Diversity Score Among Health Care Professionals in Ethiopia: A Web-Based Survey
}

\author{
Arefayne Alenko (D)' Liyew \\ Agenagnew (D)' Girma \\ Beressa (iD ${ }^{2}$ Yonas \\ Tesfaye (D) Yohannes Markos \\ Woldesenbet ${ }^{3}$ Shimelis \\ Girma (iD) \\ 'Department of Psychiatry, Institute of \\ Health, Jimma University, Jimma, Ethiopia; \\ ${ }^{2}$ Department of Public Health, School of \\ Health Sciences, Madda Walabu \\ University, Goba, Ethiopia; ${ }^{3}$ School of \\ Medicine, College of Medicine and Health \\ Sciences, Wolaitta Sodo University, \\ Wolaita Sodo, Ethiopia
}

Background: The outbreak of coronavirus disease 2019 (COVID-19) is a stressful and overwhelming situation for health care professionals (HCP), especially, who are caring for patients in the resource-limited health care settings of low-income countries. Due to the increasing number of COVID-19 cases in Ethiopia, HCPs are at risk for COVID-19-related anxiety and associated inadequate nutrition. However, the magnitude of COVID-19-related anxiety and its association with dietary diversity among HCPs is not well studied.

Objective: To assess the magnitude of COVID-19-related anxiety and its association with dietary diversity score among health care professionals in Ethiopia.

Methods: A web-based cross-sectional survey was conducted among HCPs working in university hospitals, primary hospitals, and health centers in south and southwest Ethiopia. A structured online survey questionnaire was designed on Google forms and carried out from May 15 to June 14, 2020. COVID-19-related anxiety was assessed using the coronavirus anxiety scale. Dietary diversity was measured using 9 items individual dietary diversity score. Data analysis was done using the Statistical Package for Social Sciences version 24. Multiple logistic regression was computed to identify independent factors associated with COVID-19-related anxiety. Statistical significance was set at $\mathrm{p}<0.05$.

Results: The prevalence of COVID-19-related anxiety among health care professionals was found to be $20.2 \%$. COVID-19-related anxiety is significantly associated with a low dietary diversity score $[\mathrm{AOR}=5.93(1.67,21.07)]$. The other factors which are independently associated with COVID-19-related anxiety are the presence of depression [AOR=6.98 (2.91-16.73)] and diploma educational status $[\mathrm{AOR}=0.16(0.04-0.55)]$.

Conclusion: One-fifth of the study participants were found to have probable COVID-19related anxiety. Designing a screening and intervention strategy for COVID-19-related anxiety among HCPs, particularly to those with low dietary diversity scores and depression, is recommended.

Keywords: COVID-19, anxiety, dietary diversity score, health care professionals

\section{Introduction}

The outbreak of coronavirus disease 2019 (COVID-19) is stressful for people around the world since it is declared as a pandemic by the World Health Organization (WHO). ${ }^{1}$ It is the most disrupting pandemic after the Second World War with higher morbidity and mortality around the world. ${ }^{2}$ Besides the impact of a pandemic, preventive measures such as lockdown affected the global economy, health, and social interaction. The most important aspect of health, which is
Correspondence: Arefayne Alenko Jimma University, Institute of Health, Department of Psychiatry, Faculty of Medical Science, Institute of Health, Jimma University, Jimma, Ethiopia Tel +25I 967670149

Email arefeaynealenko@gmail.com 
affected by the COVID-19 pandemic and its preventive measures such as lockdown, is mental health. The suffering and daily death reports due to COVID-19 occupied air time of all mass and social media around the world. Social media pandemic travels faster than COVID-19 and creates anxiety and panic the society. ${ }^{3}$

Many factors contribute to the disturbance of mental health during the pandemic. These are female gender, low income, living with others, higher negative feelings about consequences of the pandemic ${ }^{4-6}$ and, poor-perceived health status. ${ }^{6}$ Also, family detachment due to lockdown, and having a relative with COVID-19 infection were associated with higher levels of depression, anxiety, and stress in the general population. ${ }^{6-8}$ Furthermore, living in rural areas, having a pre-existing physical or mental illness, and longer media exposure were associated with higher odds of anxiety and depression. ${ }^{9}$

During the COVID-19 pandemic, health care workers are suffering from depression, anxiety, psychological distress, and poor sleep quality. ${ }^{6}$ Fear of getting infected by a virus while treating patients is overwhelming and causes distress among health professionals. ${ }^{10}$ Specifically, health care providers who have direct contact with patients/clients are at increased risk of psychological impact due to the relatively higher risk of encountering COVID-19. ${ }^{10,11}$ In response to the COVID-19 pandemic, health care workers taking different measures such as strictly following infection prevention and control (IPC) measures, isolate themselves, and taking different measures before joining their family. These measures probably contributed to a higher prevalence of anxiety disorder among health care workers compared to the general population during the pandemic. Feelings of uselessness since COVID-19 have no proven treatment and working with inadequate personal protective equipment (PPE), especially in developing countries, can result in significant stress. ${ }^{12}$ Besides, extensive involvement in care during death without being able to connect patients with their families, and practicing outside adequate clinical expertise can cause psychological stress. $^{13}$

Systematic review and meta-analysis conducted between April and May 2020 show about 13\% of health care professionals developed anxiety during the COVID19 pandemic. ${ }^{13}$ The prevalence of anxiety symptoms among health care workers in China is $46 \%$. In China, front-line health care workers had a higher risk of anxiety, sleep difficulty, and overall psychological problems compared with other health care workers. ${ }^{14}$ The prevalence of anxiety disorder among health care providers during the COVID-19 pandemic in Nepal is $37.3 \%$, with the majority of the participants having mild anxiety $(27.3 \%){ }^{15}$ In Oman, $25.9 \%$ of the health care workers reported moderate to severe anxiety symptoms. Of these health care workers with anxiety, $17.7 \%$ had moderate and $8.3 \%$ had severe anxiety symptoms. ${ }^{16}$ The multinational study conducted in Singapore and India reported an overall anxiety prevalence of $15.7 \%$ among health care workers. In this study, the prevalence of anxiety was $14.4 \%$ in Singapore and $17.1 \%$ in India. ${ }^{17}$ The prevalence of anxiety among health care professionals of the three COVID-19 most affected Regions in Cameroon is $42.20 \%(27.10 \%$ for moderate anxiety and $15.10 \%$ for severe anxiety). ${ }^{18} \mathrm{~A}$ high prevalence of anxiety disorder during the COVID-19 pandemic was reported among health professionals worldwide. However, there is a lack of evidence about COVID-19-related anxiety in Ethiopia.

Existing evidence highlights that dietary diversity has a significant effect on people's immunity and disease vulnerability during the COVID-19 pandemic. ${ }^{19}$ Poor dietary diversity has been linked not only with physical but also mental illnesses such as anxiety and depression. Mental illnesses can in turn affect dietary diversity due to reduced interest to eat, loss of appetite, and hopelessness. ${ }^{20}$ COVID-19 pandemic imposed a new set of challenges for the health care professionals to maintain dietary diversity to reduce susceptibility. An adequate intake of different kinds of food nutrients is predominantly vital for the maintenance of immune function during a pandemic. ${ }^{21}$ However, work-related challenges during the COVID-19 pandemic and associated psychological distress (depression, anxiety, and insomnia) can significantly impair dietary diversity or nutritional patterns among health care workers. ${ }^{18}$

Many studies assessed the prevalence of anxiety disorder among health care professionals during the COVID-19 pandemic in middle and high-income countries. However, previous studies did not explore an association between COVID-19-related anxiety and dietary diversity among health professionals. Also, there is little evidence about the prevalence of COVID-19-related anxiety among health care professions in low-income countries such as Ethiopia. As of March 29, 2021, 202,545 COVID-19 cases and 2825 COVID-19 deaths were reported in Ethiopia. A new case and death due to COVID-19 are increasing than ever. ${ }^{22}$ Despite the 
increasing burden of the pandemic and the crucial role of nutrition in boosting immunity against the coronavirus, an association between COVID-19-related anxiety and dietary diversity among health care professionals is not studied. Therefore, this study is aimed to assess the magnitude of COVID-19-related anxiety and its association with dietary diversity among health professionals in Ethiopia.

\section{Method and Materials Study Design and Setting}

A descriptive cross-sectional web-based online survey was conducted by preparing Google forms. Study participants filled online survey from May 15 to June 14, 2020. We selected a web-based online survey due to its appropriateness for full-time health care professionals, and to adhere to IPC measures of COVID-19. The study was carried out among health care professionals working in public health care facilities in south and southwest Ethiopia. University hospitals included in this study were Jimma Medical Center, Hawassa University Hospital, and Wolaita Sodo University Hospital. In addition to university hospitals, we delivered an online survey format to health professionals serving at Shenen Gibe General Hospital in Jimma town and health centers in Jimma zone, Southwest Ethiopia.

\section{Study Participants and Sample Size Determination}

Health care professionals (doctors, nurses, midwives, pharmacists, public health officers, laboratory technicians) working in the medical center, university hospitals, primary hospitals, and health centers in the south and southwest Ethiopia participated in the study. All health care professionals directly involved in patient care in the various public health facilities participated in the online survey. Health professionals who were on annual leave, doing administrative activities, and have no contact with clients, patients, and patient specimens were excluded from the study.

The minimum sample size required for this study was determined by using a single population proportion formula. The minimum sample size determination formula used is:

$$
n_{i}=\frac{\left(z_{\alpha / 2}\right)^{2} \times p(1-p)}{d^{2}}
$$

where $n_{i}$ denotes the minimum sample size, $Z \alpha / 2$ is the reliability coefficient of the standard error at a $5 \%$ level of significance $=1.96$, and $\mathrm{p}$ is the proportion of health-care providers who had moderate to severe anxiety in Oman during the COVID-19 pandemic $(p=25.9 \%) .{ }^{16}$ The calculated sample size is 295 . By adding $10 \%$ of the nonresponse rate, the total size required for this study was 325. To get a required sample size, the study participants were consecutively participated (fill online survey questionnaire) until the calculated sample size is achieved during the study period. Finally, 277 health care professionals completed online survey questionnaire.

\section{Data Collection Technique}

Data was collected using an electronically administered questionnaire by preparing Google forms. All staff were invited to participate in the online survey. Official institutional e-mail address, personal e-mail address, and social media: Facebook, Messenger, and Telegram were used to share the survey link to the staff. The URL link of the survey questionnaire is https://docs.google.com/forms/d/ 1gR7nfttTfSsFuvr6xVWqUCHr70aik3q1q3Kez7QsVR8/ edit. The objective of the study, informed consent, and maintaining confidentiality was clearly stated in the introduction section of the survey form.

\section{Data Collection Instruments}

The survey questions were prepared in the English language to get a rapid response of individuals' sociodemographic data, COVID-19-related anxiety, depression, and individual dietary diversity. The first section of the survey instrument is the socio-demographic characteristics of the participants (age, marital status, educational status, religion, and residence).

Participants' COVID-19-related anxiety was assessed using the coronavirus anxiety scale (CAS). CAS is a brief mental health screener, which is primarily developed to identify probable cases of dysfunctional anxiety associated with the COVID-19 pandemic in the last 2 weeks. CAS contains five items rated on a five-point Likert scale: $0=$ not at all, $1=$ rare (less than a day or two), $2=$ several days, $3=$ more than 7 days, $4=$ nearly every day over the last 2 weeks. It has the following items: dizziness, sleep disturbances, tonic immobility, appetite loss, and abdominal distress. The CAS can differentiate persons with and without dysfunctional anxiety with a cut score of $\geq 9(90 \%$ sensitivity and $85 \%$ specificity). ${ }^{23}$ The reliability (Cronbach's alpha) of CAS was assessed in this study and is 0.856 . 
Depression was assessed using Patient Health Questionnaire-9 (PHQ-9). PHQ-9 is a commonly used tool to assess depression in the last 2 weeks. PHQ- 9 score $\geq 10$ had a sensitivity of $88 \%$ and a specificity of $88 \%$ for major depression. ${ }^{24}$ In this study, a PHQ sum score of $\geq 10$ is screen positive for depression. Regarding the severity of depression; a total PHQ-9 score of 5-9 had minimal symptoms, 10-14 had minor depression, 15-19 had moderately severe depression and $>20$ had severe depression. ${ }^{24}$ PHQ is validated in the Ethiopian adult population with internal reliability of 0.81 and test retest reliability of $0.92 .^{25}$ The reliability (Cronbach's alpha) of PHQ-9 in this study is 0.820 . Dietary diversity was measured using 9 items individual aietary diversity score (DDS). DDS refers to the number of food groups consumed in $24 \mathrm{hrs}$ and is an indicator of a healthy diet. ${ }^{26}$ DDS score of $<4$ is Low, $4-6$ is medium and $\geq 7$ is high. ${ }^{27}$ The reliability (Cronbach's alpha) of DDS in this study is 0.620 .

\section{Data Processing and Analysis}

Data collected in online Google form was exported to MS-excel for checking and cleaning. Then, Data in MSexcel are exported to Statistical Package for Social Sciences (SPSS) version 24 for analysis. Descriptive statistics were computed and presented using frequency tables and charts. Simple binary logistic regression was fitted for each explanatory variable and those variables with $\mathrm{p}<0.25$ were fitted in multiple logistic regressions to identify independent factor/s associated with COVID19-related anxiety. Statistical significance was set at $\mathrm{p}<0.05$.

\section{Ethical Consideration}

Ethical approval was obtained from the Institute of Ethical Review Board of Jimma University (Ref. No. IRB/ 000212/2020). Information regarding the purpose of the study, voluntary participation, and risk imposed due to involvement were presented in the information section of the survey. The survey questionnaire was designed in a way the study participants only directed to the survey questions following a respondent click on a response button "Agree to participate" after reading the consent information. To ensure anonymity, information identifying the participants not included in the survey. Furthermore, the data files are stored on a password-protected computer. Therefore, this study was conducted following the Declaration of Helsinki.

\section{Results}

\section{Sociodemographic Characteristics of Study Participants}

A total of 277 health professionals participated in the study, making a response rate of $85.2 \%$. The mean (SD) age of participants was found to be 31.3 (4.80) years with minimum and maximum age of 23 and 43 years, respectively. More than half of study participants are in the age range of 30-36 years (57.1\%). The majority of the study participants were married, $163(58.8 \%)$ and having Bachelor's degree, 183 (66.1\%). More than one-third, $111(40.1 \%)$ and majority, $238(85.9 \%)$ of study participants were protestant by their religion and urban residents, respectively (Table 1).

\section{Dietary Diversity Score and Depression Among Study Participants}

Regarding dietary diversity, 89 (32.1\%) of study participants had a low dietary diversity score whereas 129 (46.6\%) had a medium dietary diversity score. Based on PHQ-9, 80 (28.9\%) study participants screened positive for depression. In terms of severity of depression, 29 $(10.5 \%)$ had moderately severe depression and 21 (7.6\%) had severe depression. Regarding the difficulty of problems or functional impairment associated with depressive symptoms, 38 (13.9\%) participants reported "Very difficult" and 22 (7.9\%) participants reported "Extremely difficult" (Table 1).

\section{Prevalence of COVID-19-Related Anxiety Among Study Participants}

Based on the coronavirus anxiety scale (CAS), the prevalence of COVID-19-related anxiety was found to be 56 (20.2\%, 95\% CI: 15.9-25.3\%) (Figure 1). The higher prevalence of COVID-19-related anxiety was observed among participants aged 30-36 year 40 (25.3, married 42 $(25.8 \%)$, urban residents $50(21 \%)$, with depression 34 $(42.5 \%)$, and having low dietary diversity score, 27 (30.3) (Table 2).

\section{Association Between COVID-19-Related Anxiety and Dietary Diversity Score}

In multivariable logistic regression analysis, COVID19-related anxiety is significantly associated with a low dietary diversity score with $\mathrm{AOR}=5.93,95 \%$ CI: 
Table I Sociodemographic Characteristics, Dietary Diversity Score, and Depression Among Study Participants in Ethiopia, 2020 $(n=277)$

\begin{tabular}{|c|c|c|c|c|c|}
\hline Variables & Categories & Frequency & Percentage (\%) & Chi-Square & P-value \\
\hline \multirow[t]{3}{*}{ Age (years) } & $23-29$ & 86 & 31 & \multirow[t]{3}{*}{7.638} & \multirow[t]{3}{*}{0.022} \\
\hline & $30-36$ & 158 & 57.1 & & \\
\hline & $37-43$ & 33 & 11.9 & & \\
\hline \multirow[t]{2}{*}{ Marital status } & Married & 163 & 58.8 & \multirow[t]{2}{*}{7.564} & \multirow[t]{2}{*}{0.006} \\
\hline & Single & 114 & 41.2 & & \\
\hline \multirow[t]{3}{*}{ Educational status } & Diploma & 76 & 27.4 & \multirow[t]{3}{*}{5.615} & \multirow[t]{3}{*}{0.060} \\
\hline & Bachelor & 183 & 66.1 & & \\
\hline & Master's degree and medical doctor & 18 & 6.5 & & \\
\hline \multirow[t]{4}{*}{ Religion } & Muslim & 73 & 26.4 & \multirow[t]{4}{*}{4.029} & \multirow[t]{4}{*}{0.258} \\
\hline & Orthodox & 83 & 30.0 & & \\
\hline & Protestant & 111 & 40.1 & & \\
\hline & Catholic & 10 & 3.6 & & \\
\hline \multirow[t]{2}{*}{ Residence } & Urban & 238 & 85.9 & \multirow[t]{2}{*}{0.657} & \multirow[t]{2}{*}{0.418} \\
\hline & Rural & 39 & 14.1 & & \\
\hline \multirow[t]{3}{*}{ Dietary diversity score } & Low & 89 & 32.1 & \multirow[t]{3}{*}{8.451} & \multirow[t]{3}{*}{0.015} \\
\hline & Medium & 129 & 46.6 & & \\
\hline & High & 59 & 21.3 & & \\
\hline \multirow[t]{2}{*}{ Depression } & Yes & 80 & 28.9 & \multirow[t]{2}{*}{34.629} & \multirow[t]{2}{*}{$<0.001$} \\
\hline & No & 197 & 71.1 & & \\
\hline \multirow[t]{5}{*}{ Severity of depression } & No & 149 & 53.8 & \multirow[t]{5}{*}{77.869} & \multirow[t]{5}{*}{$<0.001$} \\
\hline & Minimal depression & 48 & 17.3 & & \\
\hline & Minor depression & 30 & 10.8 & & \\
\hline & Moderately severe depression & 29 & 10.5 & & \\
\hline & Severe depression & 21 & 7.6 & & \\
\hline \multirow[t]{4}{*}{ Difficulty of depressive symptoms } & Not difficult at all & 126 & 45.5 & \multirow[t]{4}{*}{64.518} & \multirow[t]{4}{*}{$<0.001$} \\
\hline & Somewhat difficult & 91 & 32.9 & & \\
\hline & Very difficult & 38 & 13.9 & & \\
\hline & Extremely difficult & 22 & 7.9 & & \\
\hline \multirow[t]{2}{*}{ Depression } & Yes & 80 & 28.9 & \multirow[t]{2}{*}{34.629} & \multirow[t]{2}{*}{$<0.001$} \\
\hline & No & 197 & 7I.I & & \\
\hline
\end{tabular}

Notes: Chi-square and p-value: association between COVID-19 anxiety and respective independent variables. "Diploma" is a college level education for about 3 years after completing grade 10 in Ethiopian context.

1.67-21.07, and a p-value of 0.006. The other factors which are independently associated with COVID-19related anxiety are the presence of depression
$(\mathrm{AOR}=6.98,95 \% \mathrm{CI}: 2.91-16.73)$ and diploma educational status $\quad(\mathrm{AOR}=0.16, \quad 95 \% \quad \mathrm{CI}: \quad 0.04-0.55)$ (Table 2). 


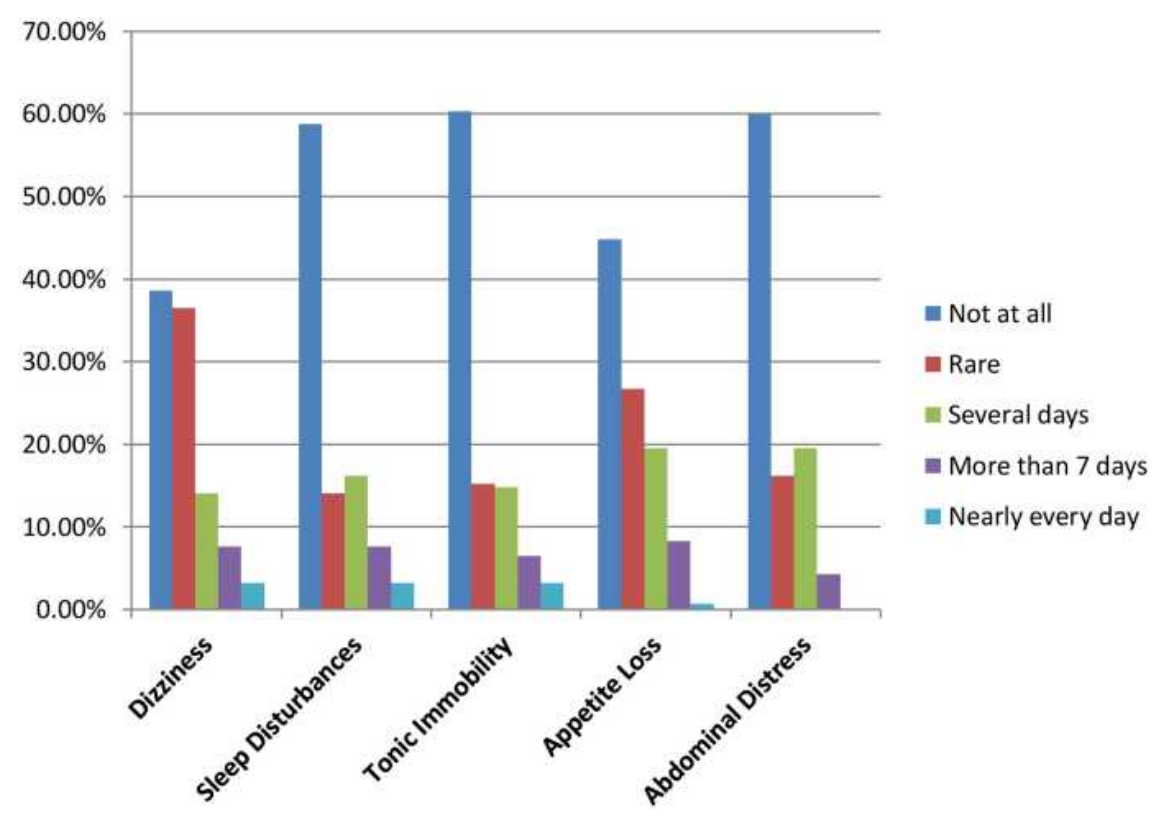

Figure I The magnitude of COVID-19-related anxiety symptoms among health professionals in Ethiopia, $2020(n=277)$.

\section{Discussion}

COVID-19-related anxiety is prevalent among health care professionals in Ethiopia. A higher prevalence of COVID19-related anxiety was observed among participants aged 30-36 years, married, urban residents, having depression, and low dietary diversity score. The odd of having COVID-19-related anxiety is six times higher among health care professionals with a low dietary diversity score. We further identified other possible risk and protective factors towards COVID-19-related anxiety. Health care providers having depression were nearly seven times more likely to develop COVID-19-related anxiety compared to health care providers not having depression. Health professionals with diploma educational status were $84 \%$ less likely to develop COVID-19-related anxiety compared to bachelor's and, master's degree and medical doctor.

This finding is in line with the study conducted in Oman among health care workers $(25.9 \%)$, of which $17 \%$ had moderate anxiety. ${ }^{28}$ Similarly, a multinational study conducted in Singapore and India reported the presence of anxiety in $15.7 \%$ of health care workers. In this study, the prevalence of anxiety was $17.1 \%$ in Indian study participants. ${ }^{17}$ The other study which supports our finding is the study conducted in three COVID-19 most affected regions of Cameroon. The study conducted in Cameron reported, $27.10 \%$ of health professionals had moderate anxiety and $15.10 \%$ had severe anxiety. ${ }^{18}$ In comparison to our finding, systematic review and meta-analysis show a lower prevalence of anxiety disorder (13\%) among health care workers during the COVID-19 pandemic. $^{13}$ This difference might be due to most included studies in systematic review and meta-analysis were conducted in China and the difference in the study period (April and May 2020).

A higher prevalence of anxiety symptoms (46\%) among health care workers was reported in China. The observed difference might be due to the inclusion of frontline workers in the study. This is evidenced by a higher risk of anxiety, sleep difficulty, and overall psychological problems among front-line health care workers compared with other health care workers. ${ }^{14}$ The odd of having COVID-19-related anxiety is nearly six times higher among health care professionals with low dietary diversity scores. This is because anxiety symptoms such as loss of appetite can impair a healthy diet. Health care workers who follow a balanced diet are strong and healthy, and thus have high immunity to the infectious and low risk to non-communicable diseases such as mental illness. Anxiety is a mood disturbance. So, disturbed mood can affect dietary diversity. Conversely, low dietary diversity affects mood and, in particular, increases anxiety levels. ${ }^{29}$ Healthier diets or diverse diet has protective effects on mental health. Individuals with mood disorders often have low dietary diversity, which is low in grains, 
Table 2 Bivariate and Multivariate Logistic Regression Analysis

\begin{tabular}{|c|c|c|c|c|c|c|}
\hline \multirow[t]{3}{*}{ Variables } & \multirow[t]{3}{*}{ Categories } & \multicolumn{2}{|c|}{$\begin{array}{l}\text { CovID-19-Related } \\
\text { Anxiety }\end{array}$} & \multirow[t]{3}{*}{$\begin{array}{l}\text { COR } \\
(95 \% \mathrm{CI})\end{array}$} & \multirow[t]{3}{*}{$\begin{array}{l}\text { AOR } \\
(95 \% \mathrm{CI})\end{array}$} & \multirow[t]{3}{*}{ P-value } \\
\hline & & Yes & No & & & \\
\hline & & $\begin{array}{l}\text { Frequency } \\
\text { (\%) }\end{array}$ & $\begin{array}{l}\text { Frequency } \\
\text { (\%) }\end{array}$ & & & \\
\hline \multirow[t]{3}{*}{ Age (years) } & $23-29$ & $9(10.5)$ & $77(89.5)$ & 1.00 & 1.00 & \\
\hline & $30-36$ & $40(25.3)$ & $118(74.7)$ & $\begin{array}{l}2.90 \\
(1.33-6.31)\end{array}$ & $\begin{array}{l}1.44 \\
(0.42-4.87)\end{array}$ & 0.559 \\
\hline & $37-43$ & $7(21.2)$ & $26(78.8)$ & $\begin{array}{l}2.30 \\
(0.78-6.80)\end{array}$ & $\begin{array}{l}1.09 \\
(0.24-4.84)\end{array}$ & 0.911 \\
\hline \multirow[t]{2}{*}{ Marital status } & Married & $42(25.8)$ & $|2|(74.2)$ & $\begin{array}{l}2.48 \\
(1.28-4.80)\end{array}$ & $\begin{array}{l}1.47 \\
(0.56-3.80)\end{array}$ & 0.428 \\
\hline & Single & $14(12.3)$ & $100(87.7)$ & 1.00 & 1.00 & \\
\hline \multirow[t]{3}{*}{ Educational status } & Diploma & $\mathrm{II}(\mathrm{I} 4.5)$ & $65(85.5)$ & $\begin{array}{l}0.53 \\
(0.26-1.10)\end{array}$ & $\begin{array}{l}0.16 \\
(0.04-0.55)\end{array}$ & $0.004 *$ \\
\hline & Bachelor & $8(44.4)$ & $10(55.5)$ & $\begin{array}{l}0.18 \\
(0.02-1.43)\end{array}$ & $\begin{array}{l}0.03 \\
(0.00-0.034)\end{array}$ & $0.005^{*}$ \\
\hline & $\begin{array}{l}\text { Master's degree and medical } \\
\text { doctor }\end{array}$ & $44(24)$ & $139(76)$ & 1.00 & 1.00 & \\
\hline \multirow[t]{2}{*}{ Residence } & Urban & $50(21)$ & $188(79)$ & $\begin{array}{l}1.46 \\
(0.58-3.68)\end{array}$ & $\begin{array}{l}1.41 \\
(0.42-4.75)\end{array}$ & 0.574 \\
\hline & Rural & $6(15.4)$ & $33(84.6)$ & 1.00 & 1.00 & \\
\hline \multirow[t]{2}{*}{ Depression } & Yes & $34(42.5)$ & $46(57.5)$ & $\begin{array}{l}5.87 \\
(3.14-11.00)\end{array}$ & $\begin{array}{l}6.98 \\
(2.91-16.73)\end{array}$ & $0.00 I^{* *}$ \\
\hline & No & $22(11.2)$ & $175(88.8)$ & 1.00 & 1.00 & \\
\hline \multirow{4}{*}{$\begin{array}{l}\text { Difficulty of depressive } \\
\text { symptoms }\end{array}$} & Not difficult at all & $23(18.3)$ & $103(81.7)$ & 1.00 & 1.00 & \\
\hline & Somewhat difficult & $24(26.4)$ & $67(73.6)$ & $\begin{array}{l}1.60 \\
(0.83-3.07)\end{array}$ & $\begin{array}{l}0.92 \\
(0.37-2.25)\end{array}$ & 0.860 \\
\hline & Very difficult & $8(2 I)$ & $30(79)$ & $\begin{array}{l}0.52 \\
(0.17-1.63)\end{array}$ & $\begin{array}{l}0.04 \\
(0.00-0.20)\end{array}$ & $0.001 * *$ \\
\hline & Extremely difficult & $5(22.7)$ & $17(77.3)$ & $\begin{array}{l}1.31 \\
(0.44-3.93)\end{array}$ & $\begin{array}{l}1.22 \\
(0.30-4.50)\end{array}$ & 0.778 \\
\hline \multirow[t]{3}{*}{ Dietary diversity score } & Low & $27(30.3)$ & $62(69.7)$ & $\begin{array}{l}2.13 \\
(0.94-4.82)\end{array}$ & $\begin{array}{l}5.93 \\
(1.67-21.07)\end{array}$ & $0.006 *$ \\
\hline & Medium & $19(14.7)$ & $110(85.3)$ & $\begin{array}{l}0.84 \\
(0.36-1.94)\end{array}$ & $\begin{array}{l}1.01 \\
(0.33-3.05)\end{array}$ & 0.986 \\
\hline & High & $10(16.9)$ & $49(83.1)$ & 1.00 & 1.00 & \\
\hline
\end{tabular}

Notes: ${ }^{*} p<0.001,{ }^{* *} p<0.05$

fruits, and vegetables but high in fat and sugar. ${ }^{30}$ Study affecting intracellular signaling and gene expression. ${ }^{31}$ shows 4 months high-fat diet can cause anxiety and depres- During the COVID-19 pandemic, an adequate intake of sive symptoms by complicating brain activity, thereby different kinds of food items is highly encouraged for the 
maintenance of the immune system. ${ }^{21}$ However, workrelated challenges and associated anxiety of encountering illness can significantly impair dietary diversity or nutritional patterns of health care workers. ${ }^{18}$ European countries that faced severe COVID-19 pandemic and strict lockdown measures were associated with inappropriate eating behaviors and increased anxiety symptoms. ${ }^{32}$ However, there is a paucity of evidence about COVID-19-related anxiety and dietary diversity score among health care workers during the pandemic.

Health care providers having depression were nearly seven times more likely to develop COVID-19 related anxiety compared to health care providers not having depression. The observed association between anxiety and depression might be due to the similarity of symptom presentation and high comorbidity. COVID-19-related anxiety symptoms such as sleep disturbance, appetite loss, and immobility or retardation are common and typical symptoms of retarded depression. ${ }^{33}$ There is also high comorbidity of depression and anxiety disorders. It is also believed that "comorbidity is the rule with anxiety and depressive disorders". ${ }^{4}$ Specifically, the comorbidity of anxiety and depression was observed at both the threshold and subthreshold diagnostic levels. ${ }^{35}$ In general, comorbidity of depressive and generalized anxiety disorders is more common than expected based on their magnitude in both primary care and the general population. ${ }^{36}$ Besides, COVID-19-related fear and anxiety about encountering a disease and associated social consequence while treating patients is a common stressful situation for both depression and anxiety among health professionals. ${ }^{10}$ Besides, health professionals with diploma educational status less likely to develop COVID-19-related anxiety compared to bachelor's, master's degree and medical doctor. There is little evidence for this finding. Therefore, further research is recommended.

\section{Strength and Limitation of the Study}

The study has several strengths. First, we addressed participants by using a web-based survey. So, there is no risk for COVID-19 infection. Second, we used validated instruments/tools to assess COVID-19-related anxiety, dietary diversity score, and depression. Third, we included participants from health facilities in different parts of the country.

A limitation of the study is that since the study primarily focused on health professionals' COVID-19related anxiety; we did not capture pre-existing anxiety disorders and other mental illnesses. The lower-reliability of the online forms is another limitation of this study. Due to the cross-sectional nature of the study, the association between different factors and COVID-19-related anxiety does not imply a cause-effect relationship.

\section{Conclusion and Recommendation}

The magnitude of COVID-19-related anxiety among health professionals is high. Health professionals with low dietary diversity scores and depression were more likely to develop COVID-19-related anxiety. Besides, health professionals with diploma educational status less likely to develop COVID-19-related anxiety. Dietary diversity and depression must be taken into account while designing a screening and intervention strategy for COVID-19-related anxiety among health professionals. Also, we recommend routine mental health and nutritional assessment of health professions for future pandemics.

\section{Abbreviations}

CAS, coronavirus anxiety scale; COVID-19, coronavirus disease 2019; DDS, dietary diversity score; HCP, health care professionals; HCW, health care worker; PHQ-9, Patient Health Questionnaire-9; PPE, personal protective equipment; SD, standard deviation; SPSS, Statistical Package for Social Sciences; WHO, World Health Organization.

\section{Acknowledgments}

The authors would like to acknowledge Jimma University and the study participants.

\section{Author Contributions}

All authors made a significant contribution to the work reported, whether that is in the conception, study design, execution, acquisition of data, analysis, and interpretation, or in all these areas; took part in drafting, revising, or critically reviewing the article; gave final approval of the version to be published; have agreed on the journal to which the article has been submitted, and agree to be accountable for all aspects of the work.

\section{Funding}

The authors did not receive any financial support for the survey work or the publication of the article.

\section{Disclosure}

The authors declare that there are no conflicts of interest to disclose. 


\section{References}

1. Committee(IASC) I-AS. IASC Interim Brief Note on Addressing Mental Health and Psychosocial Aspects of Covid-19 Outbreak. Geneva: IASC; 2020.

2. Smith SL, Raviola GJ. 'Jack be nimble, Jack be quick ... ': mental health and psychosocial response in the time of coronavirus. Global Mental Health. 2020;7.

3. Depoux A, Martin S, Karafillakis E, et al. The Pandemic of Social Media Panic Travels Faster Than the COVID-19 Outbreak. Oxford University Press; 2020.

4. Flesia L, Monaro M, Mazza C, et al. Predicting perceived stress related to the Covid-19 outbreak through stable psychological traits and machine learning models. J Clin Med. 2020;9(10).

5. Di Crosta A, Palumbo R, Marchetti D, et al. Individual differences, economic stability, and fear of contagion as risk factors for PTSD symptoms in the COVID-19 Emergency. Front Psychol. 2020;11:567367.

6. Vindegaard N, Benros ME. COVID-19 pandemic and mental health consequences: systematic review of the current evidence. Brain Behav Immun. 2020;89:531-542.

7. Mazza C, Ricci E, Biondi S, et al. A nationwide survey of psychological distress among Italian people during the COVID-19 pandemic: immediate psychological responses and associated factors. Int J Environ Res Public Health. 2020;17(9):3165.

8. Roma P, Monaro M, Colasanti M, et al. A 2-month follow-up study of psychological distress among Italian people during the COVID-19 lockdown. Int J Environ Res Public Health. 2020;17(21).

9. Wang Y, Kala MP, Jafar TH. Factors associated with psychological distress during the coronavirus disease 2019 (COVID-19) pandemic on the predominantly general population: a systematic review and meta-analysis. PLoS One. 2020;15(12):e0244630.

10. Organization WH. Mental Health and Psychosocial Considerations During the COVID-19 Outbreak. World Health Organization; 2020.

11. Control CfD, Prevention. Coronavirus Disease 2019 (COVID-19): Stress and Coping. Atlanta, EU: US Department of health \& Human Services recuperado de; 2020. Available from: https://wwwcdcgov/ coronavirus/2019-ncov/daily-life-coping/managing-stressanxietyhtml.

12. Tsamakis K, Triantafyllis AS, Tsiptsios D, et al. COVID-19 related stress exacerbates common physical and mental pathologies and affects treatment. Exp Ther Med. 2020;20(1):159-162.

13. da Silva FCT, Neto MLR. Psychological effects caused by the COVID-19 pandemic in health professionals: a systematic review with meta-analysis. Prog Neuropsychopharmacol Biol Psychiatry. 2020;110062.

14. Que J, Le Shi JD, Liu J, et al. Psychological impact of the COVID-19 pandemic on healthcare workers: a cross-sectional study in China. General Psychiatry. 2020;33(3).

15. Gupta AK, Mehra A, Niraula A, et al. Prevalence of anxiety and depression among the healthcare workers in Nepal during the COVID-19 pandemic. Asian J Psychiatr. 2020.

16. Badahdah A, Khamis F, Al Mahyijari N, et al. The mental health of health care workers in Oman during the COVID-19 pandemic. Int J Soc Psychiatry. 2020;0020764020939596.

17. Chew NW, Lee GK, Tan BY, et al. A multinational, multicentre study on the psychological outcomes and associated physical symptoms amongst healthcare workers during COVID-19 outbreak. Brain Behav Immun. 2020
18. Keubo FRN, Mboua PC, Tadongfack TD, et al. Psychological distress among health care professionals of the three COVID-19 most affected Regions in Cameroon: prevalence and associated factors. Ann Medicopsychologiques. 2020.

19. Naja F, Hamadeh R. Nutrition amid the COVID-19 pandemic: a multi-level framework for action. Eur J Clin Nutr. 2020;1-5.

20. Muscogiuri G, Barrea L, Savastano S, et al. Nutritional recommendations for CoVID-19 quarantine. Eur J Clin Nutr. 2020;1-2.

21. Butler MJ, Barrientos RM. The impact of nutrition on COVID-19 susceptibility and long-term consequences. Brain Behav Immun. 2020.

22. https://www.worldometers.info/coronavirus/?fbclid= I w A R 1 o A z C s y W c W f 7 z U e b M j C Y L S d 0 q 6 B vO6ANXWZFDQgo9d50-Yc6O9LJex6k. Accessed May 29, 2021.

23. Lee SA. Coronavirus Anxiety Scale: a brief mental health screener for COVID-19 related anxiety. Death Stud. 2020;44(7):393-401.

24. Kroenke K, Spitzer RL, Williams JB. The PHQ-9: validity of a brief depression severity measure. J Gen Intern Med. 2001;16(9):606-613.

25. Gelaye B, Williams MA, Lemma S, et al. Validity of the Patient Health Questionnaire-9 for depression screening and diagnosis in East Africa. Psychiatry Res. 2013;210(2):653-661.

26. Ty H, Dietary Diversity MK. Score: a measure of nutritional adequacy or an indicator of healthy diet? J Nutri Health Sci. 2016;3(3).

27. Kennedy G, Ballard T, Dop MC. Guidelines for Measuring Household and Individual Dietary Diversity. Food and Agriculture Organization of the United Nations; 2011.

28. Badahdah A, Khamis F, Al Mahyijari N, et al. The mental health of health care workers in Oman during the COVID-19 pandemic. Int J Soc Psychiatry. 2020;20764020939596.

29. Murphy M, Mercer JG. Diet-regulated anxiety. Int $J$ Endocrinol. 2013;2013.

30. Davison KM, Kaplan BJ. Food intake and blood cholesterol levels of community-based adults with mood disorders. BMC Psychiatry. 2012;12(1):1-8.

31. Dutheil S, Ota KT, Wohleb ES, et al. High-fat diet induced anxiety and anhedonia: impact on brain homeostasis and inflammation. Neuropsychopharmacology. 2016;41(7):1874-1887.

32. Papandreou C, Arija V, Aretouli E, et al. Comparing eating behaviours, and symptoms of depression and anxiety between Spain and Greece during the COVID-19 outbreak: cross-sectional analysis of two different confinement strategies. Eur Eating Disord Rev. 2020;28 (6):836-846.

33. Association AP. Diagnostic and Statistical Manual of Mental Disorders $\left(D S M-5^{\circledR}\right)$. American Psychiatric Pub; 2013.

34. Aina Y, Susman JL. Understanding comorbidity with depression and anxiety disorders. $J$ Am Osteopath Assoc. 2006;106(5 Suppl 2):S914

35. Preisig M, Merikangas K, Angst J. Clinical significance and comorbidity of subthreshold depression and anxiety in the community. Acta Psychiatr Scand. 2001;104(2):96-103.

36. Maier W, Falkai P. The epidemiology of comorbidity between depression, anxiety disorders and somatic diseases. Int Clin Psychopharmacol. 1999;14:S1-6. 


\section{Publish your work in this journal}

The Journal of Multidisciplinary Healthcare is an international, peerreviewed open-access journal that aims to represent and publish research in healthcare areas delivered by practitioners of different disciplines. This includes studies and reviews conducted by multidisciplinary teams as well as research which evaluates the results or conduct of such teams or healthcare processes in general. The journal covers a very wide range of areas and welcomes submissions from practitioners at all levels, from all over the world. The manuscript management system is completely online and includes a very quick and fair peer-review system. Visit http://www.dovepress.com/testimonials. php to read real quotes from published authors. 